\section{Teleoculoplastics-Teleorbit During COVID-19 Pandemic}

\section{To the Editor:}

By May 10, 2020, the coronavirus disease 2019 (COVID19) had been confirmed via PCR testing in 224,390 cases in Spain, reaching 26,621 deaths. ${ }^{1}$ Since then, we entered Phase 1 of the coronavirus deescalation process, including a relaxation of face-to-face consultations. However, from the first working day after the declaration of the state of alarm in our country (Monday, March 16, 2020) to the above-mentioned date, teleophthalmology was supposed to acquire a leading role, ${ }^{2}$ trying to attend to the strictly necessary patients, such as emergency ones, postsurgery, cases or those with ophthalmic tumors.

As members of an Oculoplastics-Orbit Unit at a tertiary referral hospital in Andalusia, the most populated Spanish Autonomous Community, we followed our authorities' instructions with regard to consultations. During the period referred above, 138 Oculoplastics-Orbit consultations had been scheduled beforehand. About $80 \%$ of them were telematically attended, mainly via phone calls and in some cases using an official application that lets patients upload photographs from their mobile phones. Nevertheless, the majority of these "teleoculoplastics-teleorbit" consultations were postponed, reaching a resolution rate of $6.31 \%$ (Table 1). Regarding the chief complaints during the state of alarm at our Unit, anophthalmic sockets, postsurgery consultations, and lacrimal disorders took up the first 3 positions, encompassing $50 \%$ of the patients (Table 2).

The main problem we found while performing telematic consultations was that our subspecialty is truly surgical, we need to "see and touch" the patient we are going to operate. This issue is very important in case of elderly patients, because they usually have limited access to technology, preferring face-to-face consultations in order to know their surgeon in person. Probably, an informed consent could be signed telematically without great difficulties, but the confidence we transmit during a visit could not be replaced by any electronic method. Evidently, "teleoculoplastics-teleorbit" consultations will never become the gold standard, but they could bring a considerable savings in visits, such as communicating Radiology or Pathology results.

In conclusion, telematic attention to patients with oculoplastic or orbital diseases could be an interesting tool to implement at our units in this new era. Despite doctors' good purposes, technical support, funds, and real involvement of authorities must be essential to guarantee success and make it efficient.

TABLE 1. Results of our Oculoplastics-Orbit consultations during the state of alarm by the coronavirus disease 2019 (COVID-19) pandemic in Spain (from March 16 to May 10,2020$)$.

\begin{tabular}{lclc}
\hline $\begin{array}{l}\text { Scheduled Oculoplastics- } \\
\text { Orbit Consultations }\end{array}$ & $\mathbf{1 3 8}(\mathbf{1 0 0 \% )})$ & & \\
\hline In-person & $20(14.49 \%)$ & Discharged & $2(10 \%)$ \\
& & Follow-up & $18(90 \%)$ \\
Telematic & $111(80.43 \%)$ & Discharged & $7(6.31 \%)$ \\
Not attended/Unanswered & $7(5.07 \%)$ & Follow-up & $104(93.69 \%)$ \\
\hline
\end{tabular}

TABLE 2. Chief complaints of our Oculoplastics-Orbit consultations during the state of alarm by the coronavirus disease 2019 (COVID-19) pandemic in Spain (from March 16 to May 10, 2020).

\begin{tabular}{lc}
\hline $\begin{array}{l}\text { Attended/answered scheduled } \\
\text { Oculoplastics-Orbit consultations }\end{array}$ & $\mathbf{1 3 1} \mathbf{( 1 0 0 \% )}$ \\
\hline Anophthalmic socket & $26(19.85 \%)$ \\
Postsurgery & $24(18.32 \%)$ \\
Lacrimal disorders & $15(11.45 \%)$ \\
Thyroid eye disease & $10(7.63 \%)$ \\
Facial palsy & $9(6.87 \%)$ \\
Conjunctival/Eyelid minor lesions & $7(5.34 \%)$ \\
Upper eyelid ptosis & $7(5.34 \%)$ \\
Mucosynechiant diseases & $7(5.34 \%)$ \\
Eyelid malpositions & $7(5.34 \%)$ \\
Meibomian gland dysfunction & $6(4.58 \%)$ \\
Blepharochalasis & $3(2.29 \%)$ \\
Idiopathic orbital inflammatory disease & $3(2.29 \%)$ \\
Congenital eyelid disorders & $3(2.29 \%)$ \\
Other orbital disorders & $3(2.29 \%)$ \\
Trichiasis & $1(0.76 \%)$ \\
\hline
\end{tabular}

\section{Antonio M. Garrido-Hermosilla, M.D. Javier Palenque-Sánchez, M.D. María Concepción Díaz-Ruiz, M.D.}

Address correspondence and reprint requests to Antonio M. Garrido-Hermosilla, M.D., Oculoplastics \& Orbit Unit, Department of Ophthalmology, Virgen Macarena University Hospital, 3 Doctor Fedriani Avenue, 41009, Seville, Spain (gaherfamily@hotmail.com)

The authors have no conflicts of interest to disclose.

\section{REFERENCES}

1. Spanish Department of National Security. Coronavirus (COVID-19)-10 de mayo 2020. Available at: https://www.dsn.gob. es/es/actualidad/sala-prensa/coronavirus-covid-19-10-mayo-2020. Accessed June 18, 2020.

2. Saleem SM, Pasquale LR, Sidoti PA, Tsai JC. Virtual ophthalmology: telemedicine in a COVID-19 era. Am J Ophthalmol 2020:S0002-9394(20)30214-2. [Epub ahead of print]

\section{A Customized Face-Shield for Ophthalmologists During COVID-19 Second Wave}

\section{To the Editor:}

SARS-CoV-2 is known to be primarily transmitted through droplet spread and contact routes; however, other routes as airborne are considered possible. ${ }^{1,2}$ There are studies reporting that SARS-CoV-2 can be detected in the conjunctival secretions of positive patients with conjunctivitis. ${ }^{3}$

Ophthalmologists are a high-risk category, because they have close contact with patients during the examination and are exposed to different potentially contaminated fluids as droplets, airborne, tears, and conjunctival secretions. As the contagion curve is rapidly increasing, personal protective equipment is essential to minimize viral spread and ensure safety. As far as eye protection is concerned, to prevent mucous membrane exposure, ophthalmologists can use goggles with good adhesion 


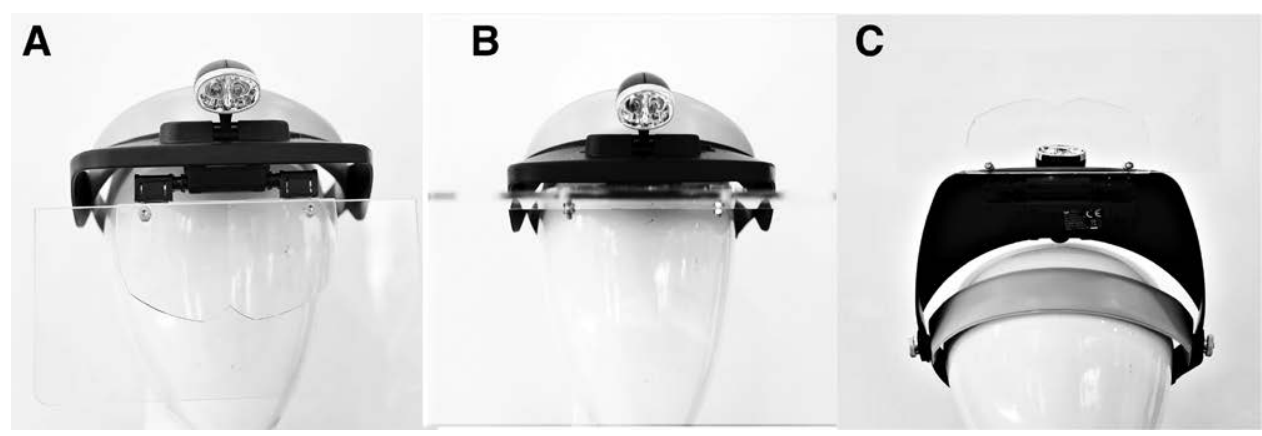

Customized face shield for ophthalmologists (A) in operative position, (B) $90^{\circ}$ tilted, and (C) $180^{\circ}$ tilted.

to the face or disposable face shields. ${ }^{4}$ However, goggles may fog easily, do not ensure splash or spray protection to other parts of the face and are uncomfortable with prolonged use..$^{5}$ Regular corrective spectacles cover only a small area and are not considered adequate eye protection. ${ }^{6}$ Face shields offer a defense from acutely expelled fluids and are commonly used as an alternative to goggles as they provide protection to a wider area of the face. ${ }^{7}$ Protection from contamination of the surface of respirators can be prevented by placing a surgical mask over it, or wearing a face shield. ${ }^{8}$ Evidence demonstrates the use of a cleanable face shield is strongly preferred to a surgical mask to reduce respirator contaminator. ${ }^{9}$ Lindsley et al. ${ }^{10}$ described respectively $96 \%$ and $92 \%$ decreases in the risk of inhalational exposure immediately after a cough for a face shield at distances of 46 and $183 \mathrm{~cm}$. However, googles and conventional face shields may prove to be uncomfortable for ophthalmologists especially when using examination instruments as slit-lamp. Therefore, to warrant performance and protection, the authors decided to customize a commercially available headband (TO-5137809, Toolcraft, Germany) that comes with LED lighting and magnification lens $(1.2 \times, 1.8 \times$, $2.5 \times$, and $3.5 \times$ ) that is suitable for different professionals as dentists, jewelry makers, etc. The authors are delighted to share practical instruction to build this shield hoping it can protect the authors' colleagues during their activity.

In particular, the authors modified the provided lenses adding, with 2 M3 12-mm screws and nuts, a 3-mm-thick, 8-cm-high, and 18-cm-wide plexiglass. Plexiglass and lenses were previously customized respectively with a $\mathrm{CO}_{2}$ laser cutter and drill press to shape 2 corresponding M3 holes. The plexiglass holes distance were $5 \mathrm{~cm}$ from the margins and $8 \mathrm{~cm}$ apart. Lenses are already fitted by the manufacturer to be attached to the headband and thus the plexiglass will follow along.

The result is a practical face shield with built-in magnification lens and LED light illumination that allows a safe close patient inspection, examination of lacrimal system, performance of Ophthalmic plastic and episcleral surgery. Moreover, it can be easily lifted up to $180^{\circ}$ to comfortably use slit lamp and other instruments, such as surgical microscopes, which may reveal uncomfortable with other eyes protection (Fig.).

During COVID-19 pandemic, ophthalmologists may benefit in their practice of this simple, economical, and customized face shield.

\section{Alberto Montericcio, M.D. Alessio Montericcio, M.D. Raffaele Raimondi, M.D.}

Correspondence: Alessio Montericcio, Via Rita Levi Montalcini 4, Pieve Emanuele, Milan, Italy (alessiomontericcio@gmail.com)

The authors have no financial or conflicts of interest to disclose.

\section{REFERENCES}

1. World Health Organization (WHO). Modes of transmission of virus causing COVID-19: implications for IPC precaution recommendations. Sci Br 2020;29:10-12.

2. Romano MR, Montericcio A, Montalbano C, et al. Facing COVID19 in ophthalmology department. Curr Eye Res 2020;45:653-658.

3. Xia J, Tong J, Liu M, et al. Evaluation of coronavirus in tears and conjunctival secretions of patients with SARS-CoV-2 infection. $J$ Med Virol. 2020;92:589-594.

4. European Centre for Disease Prevention and Control. Guidelines for Non-pharmaceutical Interventions to Reduce the Impact of COVID-19 in the EU/EEA and the UK. 24 September 2020. ECDC: Stockholm, 2020

5. Veltri K. Safety of goggles vs. face shields in the COVID-19 era. Available at: https://www.aao.org/Assets/7231d8d7-0332-406bb5b6-681558dd35d3/637215419697630000/goggles-vs-faceshieldspdf?inline=1. Accessed October 5, 2020.

6. National Health Service England. COVID-19: infection prevention and control guidance. Available at: https://www.gov.uk/government/publications/wuhan-novel-coronavirus-infection-preventionand-control. Accessed October 20, 2020.

7. Roberge RJ. Face shields for infection control: a review. J Occup Environ Hyg 2016;13:239-246.

8. Medicine I of. Reusability of Facemasks During an Influenza Pandemic: Facing the Flu. Washington, DC: The National Academies Press; 2006.

9. Erwin PC. Effect of surgical masks worn concurrently over N95 filtering facepiece respirators: extended service life versus increased user burden. J Public Health Manag Pract 2008;14:E9-E18.

10. Lindsley WG, Noti JD, Blachere FM, et al. Efficacy of face shields against cough aerosol droplets from a cough simulator. J Occup Environ Hyg 2014;11:509-518.

\section{Re: "Loss of Stimulated Tear Secretion After Orbital Reconstruction"}

\section{To the Editor:}

The authors have read with interest the paper published by Madhan and Cho, ${ }^{1}$ the first report of abnormal parasympathetic lacrimal gland innervation caused by dislocated orbital implant. Here, the authors would share 2 similar, but a bit different cases.

From January 2015 to December 2019, the authors' team has repaired 2051 cases of orbital fracture, except for those with severe craniocerebral injury or facial nerve injury. Among them, 2 cases were unable to produce tears when crying, but without sunken eyeball, diplopia, or any other complications after operation. The 2 cases had no dry eye symptom or corneal epithelial detachment. Also, the Schirmer test and the tear film break-up 